\title{
Stability of a Two-Sublattice Spin-Glass Model
}

\author{
Carlos S. O. Yokoi \\ Instituto de Física, Universidade de São Paulo, Caixa Postal 66318, 05315-970 São Paulo, SP, Brazil \\ and Francisco A. da Costa \\ Departamento de Física Teórica e Experimental, Universidade Federal do Rio Grande do Norte, \\ Caixa Postal 1641, 59072-970 Natal, RN, Brazil
}

Received on 6 October, 2003

\begin{abstract}
We study the stability of the replica-symmetric solution of a two-sublattice infinite-range spin-glass model, which can describe the transition from an antiferromagnetic to a spin-glass state. The eigenvalues associated with replica-symmetric perturbations are in general complex. The natural generalization of the usual stability condition is to require the real part of these eigenvalues to be positive. The necessary and sufficient conditions for all the roots of the secular equation to have positive real parts is given by the Hurwitz criterion. The generalized stability condition allows a consistent analysis of the phase diagram within the replica-symmetric approximation.
\end{abstract}

\section{Introduction}

The infinite-range Sherrington-Kirkpatrick (SK) model [1] for a spin glass has attracted considerable attention over the past decades [2, 3, 4]. These investigations have revealed highly non-trivial properties such as the instability of the replica-symmetric (RS) solution [5] and the replicasymmetry-breaking scheme to produce a stable solution [69]. Most studies have concentrated on situations where the exchange distributions are either symmetric or with an additional ferromagnetic interaction. More recently a twosublattice version of the SK model was introduced [10-13] to allow for antiferromagnetic interactions between different sublattices. Such extension is quite natural in view of the existence of many experimental systems such as $\mathrm{Fe}_{x} \mathrm{Mg}_{1-x} \mathrm{Cl}_{2}$ [14-16] and $\mathrm{Fe}_{x} \mathrm{Mn}_{1-x} \mathrm{TiO}_{3}$ [17, 18], which exhibit a transition from an Ising antiferromagnetic into an Ising spin glass state for a certain range of $x$ values. In contrast to the standard SK model, in the two-sublattice SK model with antiferromagnetic intersublattice interactions, the ordered (antiferromagnetic) phase extends to finite fields and the de Almeida-Thouless instability line [5] has distinct branches in the paramagnetic and antiferromagnetic phases, which do not meet at a first-order transition [10-13]. Experimental determination of the field-temperature phase diagram in $\mathrm{Fe}_{x} \mathrm{Mn}_{1-x} \mathrm{TiO}_{3}$, as well as the de AlmeidaThouless instability line [19], are in qualitative agreement with mean-field results [13].

In the previous studies of this model the stability of the RS solution against transversal fluctuations, i.e., outside the RS space, has already been investigated [10-13], and the stability against longitudinal fluctuations, i.e., inside the RS space, was also briefly considered [12]. The stability of the
RS solution against transversal fluctuations is important to establish whether replica symmetry breaking is necessary. The stability against longitudinal fluctuations, however, is also necessary to ensure the validity of RS solution. For certain parameter values of the two-sublattice SK model there may be up to three RS solutions, all of them stable against transversal fluctuations. In such a situation the analysis of the stability against longitudinal fluctuations is important for a consistent study of the phase diagram by eliminating unstable solutions.

In this work we remedy the lack of such investigation by a detailed numerical and analytical study of the eigenvalues associated with longitudinal fluctuations. Surprisingly, these eigenvalues are in general complex. It is natural to assume that stability condition should require the real part of these eigenvalues to be positive. The necessary and sufficient condition for all the roots of the secular equation to have positive real parts is given by the Hurwitz criterion. We show that this generalized stability condition allows a consistent study of the phase diagram within the RS approximation.

\section{The model}

We consider a system of $2 N$ Ising spins $S_{i}= \pm 1$ located at the sites of two identical sublattices $A$ and $B$. The interactions are described by the Hamiltonian

$$
\begin{aligned}
\mathcal{H}= & -\sum_{i \in A, j \in B} J_{i j} S_{i} S_{j}-\sum_{(i j) \in A} J_{i j}^{\prime} S_{i} S_{j} \\
& -\sum_{(i j) \in B} J_{i j}^{\prime} S_{i} S_{j}-H \sum_{i} S_{i},
\end{aligned}
$$


where the first sum is over all distinct pairs of spins belonging to different sublattices, the second and third ones refer to all distinct pairs of spins belonging to the same sublattices, and the last sum is over all spins in the two sublattices. $J_{i j}$ is the exchange interaction between spins in different sublattices, $J_{i j}^{\prime}$ is the exchange interaction between spins in the same sublattice, and $H$ is the applied magnetic field. The exchange interactions are independent, quenched, Gaussian random variables with mean values

$$
\left\langle J_{i j}\right\rangle_{J}=\frac{J_{0}}{N}, \quad\left\langle J_{i j}^{\prime}\right\rangle_{J}=\frac{J_{0}^{\prime}}{N}
$$

and variances

$$
\left\langle J_{i j}^{2}\right\rangle_{J}-\left\langle J_{i j}\right\rangle_{J}^{2}=\frac{J^{2}}{N}, \quad\left\langle J_{i j}^{\prime 2}\right\rangle_{J}-\left\langle J_{i j}^{\prime}\right\rangle_{J}^{2}=\frac{J^{\prime 2}}{N} .
$$

The mean intrasublattice interactions will always assumed to be ferromagnetic $\left(J_{0}^{\prime}>0\right)$, whereas the mean intersublattice interactions may be ferromagnetic $\left(J_{0}>0\right)$ or antiferromagnetic $\left(J_{0}<0\right)$.

The standard approach to compute the quenched average is to introduce $n$ non-interacting replicas $\alpha=1,2, \ldots, n$ of the system, calculate the annealed averages and then take the limit $n \rightarrow 0[2,4]$. In this replica method the free energy per spin $f$ is given by

$$
f=\frac{1}{2 \beta} \lim _{n \rightarrow 0} \frac{1}{n} \phi, \quad \phi=-\lim _{N \rightarrow \infty} \frac{1}{N} \ln \left\langle Z^{n}\right\rangle_{J},
$$

where $\beta=1 / k_{\mathrm{B}} T$ and $Z^{n}$ is the partition function of $n$ replicas of the system. Performing the average of $Z^{n}$ over the random couplings we find

$$
\begin{aligned}
\left\langle Z^{n}\right\rangle_{J}= & \operatorname{Tr} \exp -N\left\{-\frac{\beta^{2} J^{2} n}{2}+\beta J_{0}^{\prime} \frac{n}{N}-\frac{\beta^{2} J^{\prime 2} n}{2}\left(1-\frac{n}{N}\right)-\beta H \sum_{\alpha}\left(m_{A}^{\alpha}+m_{B}^{\alpha}\right)\right. \\
& -\beta J_{0} \sum_{\alpha} m_{A}^{\alpha} m_{B}^{\alpha}-\frac{\beta J_{0}^{\prime}}{2} \sum_{\alpha}\left[\left(m_{A}^{\alpha}\right)^{2}+\left(m_{B}^{\alpha}\right)^{2}\right]-\beta^{2} J^{2} \sum_{(\alpha \beta)} q_{A}^{\alpha \beta} q_{B}^{\alpha \beta} \\
& \left.-\frac{\beta^{2} J^{\prime 2}}{2} \sum_{(\alpha \beta)}\left[\left(q_{A}^{\alpha \beta}\right)^{2}+\left(q_{B}^{\alpha \beta}\right)^{2}\right]\right\},
\end{aligned}
$$

where $(\alpha \beta)$ denotes distinct pairs of replicas and we have introduced the sublattice magnetization and sublattice overlap function of the replicas,

$$
m_{X}^{\alpha}=\frac{1}{N} \sum_{i \in X} S_{i}^{\alpha}, \quad q_{X}^{\alpha \beta}=\frac{1}{N} \sum_{i \in X} S_{i}^{\alpha} S_{i}^{\beta}, \quad(X=A, B) .
$$

The trace over the spin variables in (5) can be performed by taking into account the constraints (6) by means of the identities

$$
1=\int_{-\infty}^{\infty} d m_{X}^{\alpha} \int_{-i \infty}^{i \infty} \frac{N d \lambda_{X}^{\alpha}}{2 \pi i} \exp \left[-N \lambda_{X}^{\alpha}\left(m_{X}^{\alpha}-\frac{1}{N} \sum_{i \in X} S_{i}^{\alpha}\right)\right] \quad(X=A, B),
$$

and

$$
1=\int_{-\infty}^{\infty} d q_{X}^{\alpha \beta} \int_{-i \infty}^{i \infty} \frac{N d \lambda_{X}^{\alpha \beta}}{2 \pi i} \exp \left[-N \lambda_{X}^{\alpha \beta}\left(q_{X}^{\alpha \beta}-\frac{1}{N} \sum_{i \in X} S_{i}^{\alpha} S_{i}^{\beta}\right)\right] \quad(X=A, B) .
$$

We then obtain

$$
\begin{aligned}
\left\langle Z^{n}\right\rangle_{J}= & \prod_{\alpha} \int_{-\infty}^{\infty} d m_{A}^{\alpha} \int_{-i \infty}^{i \infty} \frac{N d \lambda_{A}^{\alpha}}{2 \pi i} \int_{-\infty}^{\infty} d m_{B}^{\alpha} \int_{-i \infty}^{i \infty} \frac{N d \lambda_{B}^{\alpha}}{2 \pi i} \prod_{(\alpha \beta)} \int_{-\infty}^{\infty} d q_{A}^{\alpha \beta} \int_{-i \infty}^{i \infty} \frac{N d \lambda_{A}^{\alpha \beta}}{2 \pi i} \\
& \times \int_{-\infty}^{\infty} d q_{B}^{\alpha \beta} \int_{-i \infty}^{i \infty} \frac{N d \lambda_{B}^{\alpha \beta}}{2 \pi i} \exp \left[-N \phi\left(m_{A}^{\alpha}, m_{B}^{\alpha}, q_{A}^{\alpha \beta}, q_{B}^{\alpha \beta} ; \lambda_{A}^{\alpha}, \lambda_{B}^{\alpha}, \lambda_{A}^{\alpha \beta}, \lambda_{B}^{\alpha \beta}\right)\right],
\end{aligned}
$$

where

$$
\begin{aligned}
\phi= & -\frac{\beta^{2} J^{2} n}{2}+\beta J_{0}^{\prime} \frac{n}{N}-\frac{\beta^{2} J^{\prime 2} n}{2}\left(1-\frac{n}{N}\right)-\beta H \sum_{\alpha}\left(m_{A}^{\alpha}+m_{B}^{\alpha}\right)-\beta J_{0} \sum_{\alpha} m_{A}^{\alpha} m_{B}^{\alpha} \\
& -\frac{\beta J_{0}^{\prime}}{2} \sum_{\alpha}\left[\left(m_{A}^{\alpha}\right)^{2}+\left(m_{B}^{\alpha}\right)^{2}\right]-\beta^{2} J^{2} \sum_{(\alpha \beta)} q_{A}^{\alpha \beta} q_{B}^{\alpha \beta}-\frac{\beta^{2} J^{\prime 2}}{2} \sum_{(\alpha \beta)}\left[\left(q_{A}^{\alpha \beta}\right)^{2}+\left(q_{B}^{\alpha \beta}\right)^{2}\right] \\
& +\sum_{\alpha}\left(\lambda_{A}^{\alpha} m_{A}^{\alpha}+\lambda_{B}^{\alpha} m_{B}^{\alpha}\right)+\sum_{(\alpha \beta)}\left(\lambda_{A}^{\alpha \beta} q_{A}^{\alpha \beta}+\lambda_{B}^{\alpha \beta} q_{B}^{\alpha \beta}\right)-\ln \operatorname{Tr} \exp \overline{\mathcal{H}}_{A}-\ln \operatorname{Tr} \exp \overline{\mathcal{H}}_{B},
\end{aligned}
$$


with $\overline{\mathcal{H}}_{A}$ and $\overline{\mathcal{H}}_{B}$ denoting the "effective sublattice Hamiltonians",

$$
\overline{\mathcal{H}}_{X}=\sum_{\alpha} \lambda_{X}^{\alpha} S^{\alpha}+\sum_{(\alpha \beta)} \lambda_{X}^{\alpha \beta} S^{\alpha} S^{\beta} \quad(X=A, B) .
$$

In the limit of large $N$ the integrations over the $\lambda$ variables in (9) can be performed by the saddle-point method. The saddle point is given by

$$
m_{X}^{\alpha}=\frac{\operatorname{Tr} S^{\alpha} \exp \overline{\mathcal{H}}_{X}}{\operatorname{Tr} \exp \overline{\mathcal{H}}_{X}}, \quad q_{X}^{\alpha \beta}=\frac{\operatorname{Tr} S^{\alpha} S^{\beta} \exp \overline{\mathcal{H}}_{X}}{\operatorname{Tr} \exp \overline{\mathcal{H}}_{X}}, \quad(X=A, B) .
$$

These equations determine $\lambda$ variables in terms of $m$ and $q$ variables. The remaining integrations over the $m$ and $q$ variables in (9) can be performed by the Laplace method in the limit of large $N$. The stationary-point equations are given by

$$
\lambda_{X}^{\alpha}=\beta H+\beta J_{0}^{\prime} m_{X}^{\alpha}+\beta J_{0} m_{X}^{\alpha}, \quad \lambda_{X}^{\alpha \beta}=\beta^{2} J^{\prime 2} q_{X}^{\alpha \beta}+\beta^{2} J^{2} q_{X}^{\alpha \beta}, \quad(X=A, B),
$$

where $\bar{X}$ is the sublattice complementary to $X$, i.e., if $X=A$ then $\bar{X}=B$, and vice versa. Substituting these results in the expression of $\phi$ given by Eq. (10) we find

$$
\begin{aligned}
\phi= & -\frac{\beta^{2} n}{2}\left(J^{2}+J^{\prime 2}\right)+\beta J_{0} \sum_{\alpha} m_{A}^{\alpha} m_{B}^{\alpha}+\frac{\beta J_{0}^{\prime}}{2} \sum_{\alpha}\left[\left(m_{A}^{\alpha}\right)^{2}+\left(m_{B}^{\alpha}\right)^{2}\right]+\beta^{2} J^{2} \sum_{(\alpha \beta)} q_{A}^{\alpha \beta} q_{B}^{\alpha \beta} \\
& +\frac{\beta^{2} J^{\prime 2}}{2} \sum_{(\alpha \beta)}\left[\left(q_{A}^{\alpha \beta}\right)^{2}+\left(q_{B}^{\alpha \beta}\right)^{2}\right]-\ln \operatorname{Tr} \exp \overline{\mathcal{H}}_{A}-\ln \operatorname{Tr} \exp \overline{\mathcal{H}}_{B},
\end{aligned}
$$

where we have discarded terms that vanish in the limit of large $N$. Analogously, the effective sublattice Hamiltonians (11) become

$$
\overline{\mathcal{H}}_{X}=\beta \sum_{\alpha}\left(H+J_{0}^{\prime} m_{X}^{\alpha}+J_{0} m_{\frac{\alpha}{X}}^{\alpha} S^{\alpha}+\beta^{2} \sum_{(\alpha \beta)}\left(J^{\prime 2} q_{X}^{\alpha \beta}+J^{2} q_{\bar{X}}^{\alpha \beta}\right) S^{\alpha} S^{\beta}, \quad(X=A, B) .\right.
$$

To evaluate the general expressions obtained thus far it is necessary to impose some structure on $m$ and $q$ variables. The simplest assumption corresponds to the RS solution[2, 4] obtained by assuming order parameters independent of replica indices,

$$
m_{X}^{\alpha}=m_{X}, \quad q_{X}^{\alpha \beta}=q_{X}, \quad(X=A, B) .
$$

Proceeding in the usual way [2, 4], one finds that the saddle-point equations (12) and stationary-point equations (13) give the equations of state

$$
m_{X}=\left\langle\tanh H_{X}\right\rangle, \quad q_{X}=\left\langle\tanh ^{2} H_{X}\right\rangle, \quad(X=A, B),
$$

where

$$
H_{X}=\beta\left(H+J_{0}^{\prime} m_{X}+J_{0} m_{\bar{X}}+\sqrt{J^{\prime 2} q_{X}+J^{2} q_{\bar{X}}} x\right), \quad(X=A, B),
$$

and the brackets without subscript $\langle\cdots\rangle$ denote Gaussian averages,

$$
\langle\cdots\rangle=\int_{-\infty}^{\infty} \frac{d x}{\sqrt{2 \pi}} e^{-x^{2} / 2}(\cdots)
$$

Analogously, the free energy per spin (4) becomes

$$
\begin{aligned}
f= & -\frac{\beta J^{2}}{4}\left(1-q_{A}\right)\left(1-q_{B}\right)-\frac{\beta J^{\prime 2}}{8}\left[\left(1-q_{A}\right)^{2}+\left(1-q_{B}\right)^{2}\right]+\frac{J_{0}}{2} m_{A} m_{B}+\frac{J_{0}^{\prime}}{4}\left(m_{A}^{2}+m_{B}^{2}\right) \\
& -\frac{1}{2 \beta}\left\langle\ln 2 \cosh H_{A}\right\rangle-\frac{1}{2 \beta}\left\langle\ln 2 \cosh H_{B}\right\rangle .
\end{aligned}
$$

\section{The stability of replica-symmetric solution}

The validity of the RS solution (17) rests on the applicability of Laplace method used to perform the integrations over $m$ and $q$ variables for large $N$. The integral converges only if the stationary point (13) is a minimum of $\phi$, i.e., only if the eigenvalues of the Hessian matrix formed by the second derivatives of the function $\phi$ given by equation (10) with 
respect to the $m$ and $q$ variables are all positive. We can equivalently consider $\phi$ as a function of $\lambda$ variables, related to $m$ and $q$ variables by means of Eq. (12). We will follow the latter approach because it leads to simpler calculations. The Hessian is a $[n(n+1) / 2] \times[n(n+1) / 2]$ matrix whose elements are $2 \times 2$ matrices given by

$$
\mathbf{G}^{\alpha \beta}=\left(\begin{array}{cc}
G_{A A}^{\alpha \beta} & G_{A B}^{\alpha \beta} \\
G_{B A}^{\alpha \beta} & G_{B B}^{\alpha \beta}
\end{array}\right), \mathbf{G}^{\alpha(\beta \gamma)}=\left(\begin{array}{ll}
G_{A A}^{\alpha(\beta \gamma)} & G_{A B}^{\alpha(\beta \gamma)} \\
G_{B A}^{\alpha(\beta \gamma)} & G_{B B}^{\alpha(\beta \gamma)}
\end{array}\right), \mathbf{G}^{(\alpha \beta)(\gamma \delta)}=\left(\begin{array}{ll}
G_{A A}^{(\alpha \beta)(\gamma \delta)} & G_{A B}^{(\alpha \beta)(\gamma \delta)} \\
G_{B A}^{(\alpha \beta)(\gamma \delta)} & G_{B B}^{(\alpha \beta)(\gamma \delta)}
\end{array}\right)
$$

where

$$
G_{X Y}^{\alpha \beta}=\frac{\partial^{2} \phi}{\partial \lambda_{X}^{\alpha} \partial \lambda_{Y}^{\beta}}, \quad G_{X Y}^{\alpha(\beta \gamma)}=\frac{\partial^{2} \phi}{\partial \lambda_{X}^{\alpha} \partial \lambda_{Y}^{(\beta \gamma)}}, \quad G_{X Y}^{(\alpha \beta)(\gamma \delta)}=\frac{\partial^{2} \phi}{\partial \lambda_{X}^{\alpha \beta} \partial \lambda_{Y}^{\gamma \delta}} \quad(X, Y=A, B) .
$$

At the stationary point of the RS solution (17) there are seven different types of $2 \times 2$ elements of the Hessian matrix. We denote these elements by [5]

$$
\begin{aligned}
& \mathbf{G}^{\alpha \alpha}=\mathbf{A}, \quad \mathbf{G}^{\alpha \beta}=\mathbf{B}, \quad \mathbf{G}^{\alpha(\alpha \beta)}=\mathbf{C}, \quad \mathbf{G}^{(\alpha \beta) \alpha}=\widetilde{\mathbf{C}}, \quad \mathbf{G}^{\alpha(\beta \gamma)}=\mathbf{D}, \\
& \mathbf{G}^{(\alpha \beta) \gamma}=\widetilde{\mathbf{D}} \\
& \mathbf{G}^{(\alpha \beta)(\alpha \beta)}=\mathbf{P} \text {, } \\
& \mathbf{G}^{(\alpha \beta)(\alpha \gamma)}=\mathbf{Q} \\
& \mathbf{G}^{(\alpha \beta)(\gamma \delta)}=\mathbf{R} \text {, }
\end{aligned}
$$

where the indices $\alpha, \beta, \gamma$ and $\delta$ are all distinct and the tilde denotes the transpose of the matrix. We do not quote the lengthy expressions for these elements because only their linear combinations are needed in the calculation of the eigenvalues.

The eigenvalues of the Hessian matrix can now be determined by finding the eigenvectors that divide the space into orthogonal subspaces closed to the permutation operation. The procedure are analogous to the case of the SK model [5] except that now the elements of the Hessian ma- trix are $2 \times 2$ matrices (23). These eigenvectors are [20]: $n(n-3)$ transversal or replicon eigenvectors depending on two replica indices, $4(n-1)$ anomalous eigenvalues depending on a single replica index, and 4 longitudinal eigenvectors independent of replica indices.

The eigenvalues associated with the transversal eigenvectors are found to be the eigenvalues of the $2 \times 2$ matrix

$$
\mathbf{T}=\mathbf{P}-\mathbf{2 Q}+\mathbf{R}
$$

with elements

$$
\begin{aligned}
& T_{11}=\left(1-2 q_{A}+r_{A}\right)-\left(\beta J^{\prime}\right)^{2}\left(1-2 q_{A}+r_{A}\right)^{2} \\
& T_{12}=T_{21}=-(\beta J)^{2}\left(1-2 q_{A}+r_{A}\right)\left(1-2 q_{B}+r_{B}\right) \\
& T_{22}=\left(1-2 q_{B}+r_{B}\right)-\left(\beta J^{\prime}\right)^{2}\left(1-2 q_{B}+r_{B}\right)^{2}
\end{aligned}
$$

where

$$
t_{X}=\left\langle\tanh ^{3} H_{X}\right\rangle, \quad r_{X}=\left\langle\tanh ^{4} H_{X}\right\rangle, \quad(X=A, B) .
$$

The necessary and sufficient condition for all the eigenvalues to be positive are

$$
T_{11}+T_{22}>0 \quad \text { and } \quad T_{11} T_{22}-T_{12}^{2}>0,
$$

which are equivalent to the conditions

$$
\begin{aligned}
T_{1}= & 2-\left(\beta J^{\prime}\right)^{2}\left(1-2 q_{A}+r_{A}\right)-\left(\beta J^{\prime}\right)^{2}\left(1-2 q_{B}+r_{B}\right)>0 \\
T_{2}= & {\left[1-\left(\beta J^{\prime}\right)^{2}\left(1-2 q_{A}+r_{A}\right)\right]\left[1-\left(\beta J^{\prime}\right)^{2}\left(1-2 q_{B}+r_{B}\right)\right] } \\
& -(\beta J)^{4}\left(1-2 q_{A}+r_{A}\right)\left(1-2 q_{B}+r_{B}\right)>0
\end{aligned}
$$

in agreement with previous studies [10, 13]. A RS solution satisfying these conditions will be called transversally (T) stable, and $\mathrm{T}$ unstable otherwise.

The eigenvalues associated with anomalous and longitudinal eigenvectors are the same in the limit $n \rightarrow 0$. They are found to be the eigenvalues of the $4 \times 4$ matrix

$$
\mathbf{L}=\left(\begin{array}{ll}
\mathbf{A}-\mathbf{B} & \mathbf{D}-\mathbf{C} \\
2 \widetilde{\mathbf{C}}-2 \widetilde{\mathbf{D}} & \mathbf{P}-4 \mathbf{Q}+3 \mathbf{R}
\end{array}\right)
$$


where

$$
\begin{aligned}
& L_{11}=\left(1-q_{A}\right)-\beta J_{0}^{\prime}\left(1-q_{A}\right)^{2}+2\left(\beta J^{\prime}\right)^{2}\left(t_{A}-m_{A}\right)^{2}, \\
& L_{22}=\left(1-q_{B}\right)-\beta J_{0}^{\prime}\left(1-q_{B}\right)^{2}+2\left(\beta J^{\prime}\right)^{2}\left(t_{B}-m_{B}\right)^{2}, \\
& L_{12}=L_{21}=-\beta J_{0}\left(1-q_{A}\right)\left(1-q_{B}\right)+2(\beta J)^{2}\left(t_{A}-m_{A}\right)\left(t_{B}-m_{B}\right), \\
& L_{13}=-\frac{1}{2} L_{31}=\left(t_{A}-m_{A}\right)\left[1-\beta J_{0}^{\prime}\left(1-q_{A}\right)-\left(\beta J^{\prime}\right)^{2}\left(1-4 q_{A}+3 r_{A}\right)\right], \\
& L_{24}=-\frac{1}{2} L_{42}=\left(t_{B}-m_{B}\right)\left[1-\beta J_{0}^{\prime}\left(1-q_{B}\right)-\left(\beta J^{\prime}\right)^{2}\left(1-4 q_{B}+3 r_{B}\right)\right], \\
& L_{14}=-\frac{1}{2} L_{41}=-\beta J_{0}\left(t_{B}-m_{B}\right)\left(1-q_{A}\right)-(\beta J)^{2}\left(t_{A}-m_{A}\right)\left(1-4 q_{B}+3 r_{B}\right), \\
& L_{23}=-\frac{1}{2} L_{32}=-\beta J_{0}\left(t_{A}-m_{A}\right)\left(1-q_{B}\right)-(\beta J)^{2}\left(t_{B}-m_{B}\right)\left(1-4 q_{A}+3 r_{A}\right), \\
& L_{33}=\left(1-4 q_{A}+3 r_{A}\right)\left[1-\left(\beta J^{\prime}\right)^{2}\left(1-4 q_{A}+3 r_{A}\right)\right]+2 \beta J_{0}^{\prime}\left(t_{A}-m_{A}\right)^{2}, \\
& L_{44}=\left(1-4 q_{B}+3 r_{B}\right)\left[1-\left(\beta J^{\prime}\right)^{2}\left(1-4 q_{B}+3 r_{B}\right)\right]+2 \beta J_{0}^{\prime}\left(t_{B}-m_{B}\right)^{2}, \\
& L_{34}=L_{43}=-(\beta J)^{2}\left(1-4 q_{A}+3 r_{A}\right)\left(1-4 q_{B}+3 r_{B}\right)+2 \beta J_{0}\left(t_{A}-m_{A}\right)\left(t_{B}-m_{B}\right) .
\end{aligned}
$$

The characteristic equation has the form

$$
\lambda^{4}-a_{1} \lambda^{3}+a_{2} \lambda^{2}-a_{3} \lambda+a_{4}=0,
$$

where the coefficients $a_{n}$ are $n$-th order traces of the matrix L. A numerical study of equation (43) shows that the eigenvalues are complex for some values of the parameters of the model, in contrast with one-sublattice SK model in which the anomalous and longitudinal eigenvalues never become complex [5]. Even though the Hessian matrix (21) for $n>1$ is real and symmetric, in the limit $n \rightarrow 0$ there is no guarantee that the eigenvalues will be real. In fact, complex longitudinal and anomalous eigenvalues also arise in the spin 1 one-sublattice infinite-range spin-glass model with crystalfield anisotropy [21, 22]. In general, therefore, the stability condition should require the real part of the eigenvalues to be positive. According to the Hurwitz criterion [23], the necessary and sufficient condition for all the roots of equation (43) to have positive real parts are

$$
\begin{aligned}
& D_{1}=a_{1}>0, \quad D_{2}=\left|\begin{array}{cc}
a_{1} & a_{3} \\
1 & a_{2}
\end{array}\right|=a_{1} a_{2}-a_{3}>0, \\
& D_{3}=\left|\begin{array}{ccc}
a_{1} & a_{3} & 0 \\
1 & a_{2} & a_{4} \\
0 & a_{1} & a_{3}
\end{array}\right|=a_{3} D_{2}-a_{1}^{2} a_{4}>0, \quad D_{4}=\left|\begin{array}{cccc}
a_{1} & a_{3} & 0 & 0 \\
1 & a_{2} & a_{4} & 0 \\
0 & a_{1} & a_{3} & 0 \\
0 & 1 & a_{2} & a_{4}
\end{array}\right|=a_{4} D_{3}>0 .
\end{aligned}
$$

These condition are equivalent to the following four conditions:

$$
\begin{aligned}
& L_{1}=a_{1}>0, \\
& L_{2}=D_{2}=a_{1} a_{2}-a_{3}>0, \\
& L_{3}=D_{3}=a_{1} a_{2} a_{3}-a_{3}^{2}-a_{4} a_{1}^{2}>0, \\
& L_{4}=a_{4}>0 .
\end{aligned}
$$

A RS solution satisfying these conditions will be called longitudinally (L) stable, and L unstable otherwise.

\section{Results of the stability analysis}

In this section we present the results of the stability analysis of the RS solution for different values of the parameters of the model. Since the Hamiltonian (1) is invariant under the simultaneous transformations

$$
H \rightarrow-H, \quad S_{i} \rightarrow-S_{i},
$$

it is sufficient to consider fields $H \geq 0$. For $H=0$ only one of the two solutions related by the global inversion symmetry has to be considered. 


\subsection{Zero applied field}

\subsubsection{Ferromagnetic intersublattice interaction}

In zero applied field $(H=0)$ and ferromagnetic intersublattice interactions $\left(J_{0}>0\right)$ the solutions of the set of equations (17) are of the form

$$
m_{A}=m_{B}=m, \quad q_{A}=q_{B}=q .
$$

Three types of solutions are possible:

- Paramagnetic (P) solution: $m=0, q=0$.

- Spin Glass (SG) solution: $q>0, m=0$.

- Ferromagnetic (F) solution: $q>0, m>0$.

Fig. 1 shows the lines delimiting the regions where different types of solutions can be found in the plane of temperature versus $J_{0}+J_{0}^{\prime}$.

The $\mathrm{P}$ solution is always possible. However it is $\mathrm{L}$ stable only above the line (b) and the left portion of line (a), and T stable above line (a). The L instability of $\mathrm{P}$ solution occurs due to the violation of the condition (49), which is given in the case of P solution by

$$
\begin{gathered}
L_{4}=\left[1-\beta^{2}\left(J^{\prime 2}-J^{2}\right)\right]\left[1-\beta^{2}\left(J^{\prime 2}+J^{2}\right)\right] \\
{\left[1-\beta\left(J_{0}^{\prime}-J_{0}\right)\right]\left[1-\beta\left(J_{0}^{\prime}+J_{0}\right)\right]>0 .}
\end{gathered}
$$

For $\left(J_{0}+J_{0}^{\prime}\right) / \sqrt{J^{2}+J^{\prime 2}} \leq 1 / 2$, the second factor of in (52) becomes negative below line (a). Thus the left portion of line (a) is determined by

$$
\beta^{2}\left(J^{\prime 2}+J^{2}\right)=1
$$

On the other hand, for $\left(J_{0}+J_{0}^{\prime}\right) / \sqrt{J^{2}+J^{\prime 2}}>1 / 2$ the fourth factor in (52) becomes negative below line (b). Thus the equation for line (b) is

$$
\beta\left(J_{0}^{\prime}+J_{0}\right)=1 \text {. }
$$

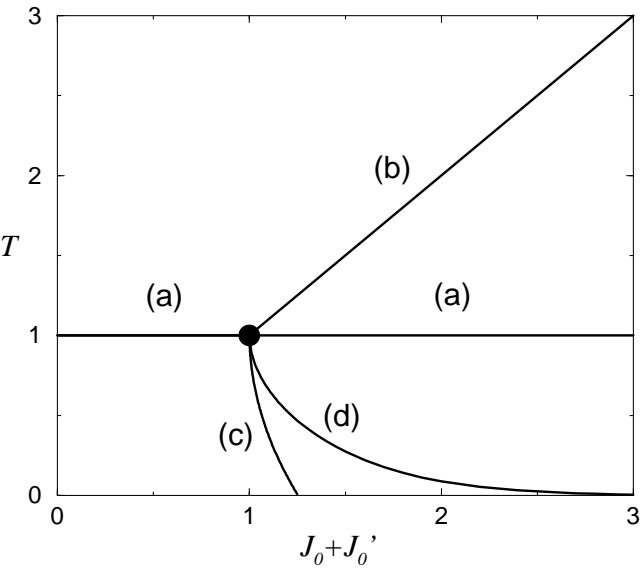

Figure 1. Regions of the zero-field phase diagram where different types of solution are possible. For ferromagnetic intersublattice interaction $\left(J_{0}>0\right)$, the $\mathrm{P}$ solution is $\mathrm{L}$ stable above line (b) and the left portion of line (a), the F solution between lines (b) and (c), and SG solution between the left portion of line (a) and line (c). The $\mathrm{P}$ solution is $\mathrm{T}$ stable above line (a), the F solution between lines (b) and (d). The SG solution is T unstable between the left side of line (a) and line (c), and F solution between lines (c) and (d). For antiferromagnetic intersublattice interaction $\left(J_{0}<0\right)$ the $\mathrm{F}$ solution is replaced by AF solution and the label of horizontal axis by $-J_{0}+J_{0}^{\prime}$. The temperature and energy units in the axis are such that $\sqrt{J^{2}+J^{\prime 2}}=1$ and $k_{B}=1$.

The $\mathrm{T}$ instability of the $\mathrm{P}$ solution is due to the violation of the condition (31), which is given in the case of $\mathrm{P}$ solution by

$$
T_{2}=\left[1-\beta^{2}\left(J^{\prime 2}-J^{2}\right)\right]\left[1-\beta^{2}\left(J^{\prime 2}+J^{2}\right)\right]>0 .
$$

The second factor in (55) becomes negative below line (a) for all values of $J_{0}+J_{0}^{\prime}$. Thus line (a) is given by equation (53) for all $J_{0}+J_{0}^{\prime}$. The $\mathrm{T}$ and $\mathrm{L}$ instabilities of the $\mathrm{P}$ solution occur simultaneously on the line (a) for $\left(J_{0}+J_{0}^{\prime}\right) / \sqrt{J^{2}+J^{\prime 2}} \leq 1 / 2$.

The SG solution is possible only below line (a). It is $\mathrm{T}$ unstable throughout this region and $\mathrm{L}$ stable to the left of line (c). The L instability of the SG solution occurs due to the violation of the condition (49), which is given in the case of SG solution by

$$
\begin{aligned}
L_{4}=(1-q)^{2}(1-4 q+3 r)^{2} \quad & {\left[1-\beta^{2}\left(J^{\prime 2}-J^{2}\right)(1-4 q+3 r)\right]\left[1-\beta^{2}\left(J^{\prime 2}+J^{2}\right)(1-4 q+3 r)\right] } \\
& \times\left[1-\beta\left(J_{0}^{\prime}-J_{0}\right)(1-q)\right]\left[1-\beta\left(J_{0}^{\prime}+J_{0}\right)(1-q)\right]>0 .
\end{aligned}
$$

For $\left(J_{0}+J_{0}^{\prime}\right) / \sqrt{J^{2}+J^{\prime 2}}>1 / 2$ the last factor in (56) becomes negative to the left of line (c). Thus the equation determining line $(\mathrm{c})$ is

$$
\beta\left(J_{0}^{\prime}+J_{0}\right)(1-q)=1 .
$$

The F solution is possible only between lines (b) and (c). It is L stable throughout this region but T stable only above line (d). The T instability of the F solution occurs due to the violation of the condition (31), which is given in the case of $\mathrm{F}$ solution by

$$
T_{2}=\left[1-\beta^{2}\left(J^{\prime 2}-J^{2}\right)(1-2 q+3 r)\right]\left[1-\beta^{2}\left(J^{\prime 2}+J^{2}\right)(1-2 q+3 r)\right]>0 .
$$


For $\left(J_{0}+J_{0}^{\prime}\right) / \sqrt{J^{2}+J^{\prime 2}}>1 / 2$ the second factor in (58) becomes negative below line (d). Thus line (d) is described by equation

$$
\beta^{2}\left(J^{\prime 2}+J^{2}\right)(1-2 q+3 r)=1 .
$$

Rejecting solutions that are $\mathrm{L}$ unstable, we conclude that the $\mathrm{P}$ phase is located above lines (b) and left portion of line (a), the SG phase between the left portion of line (a) and line (c), and finally the F phase between lines (b) and (c). The SG phase, and the F phase between lines (c) and (d), are $\mathrm{T}$ unstable, indicating the need for a replica-symmetrybreaking solution in this region. The transition line (c) will change to a vertical line if such a solution is considered [24]. We mention that, as should be expected, in the case $J_{0}=0$ and $J=0$, or $J_{0}^{\prime}=0$ and $J^{\prime}=0$, these results reduce to those of one-sublattice SK model [2, 4].

\subsubsection{Antiferromagnetic intersublattice interaction}

The Hamiltonian (1) in zero applied field $(H=0)$ is invariant under simultaneous transformations

$$
J_{0} \longrightarrow-J_{0}, \quad S_{i} \longrightarrow-S_{i} \quad(i \in B) .
$$

In fact, we can check explicitly that all the expressions for the RS solution, including those of stability conditions, are invariant under simultaneous transformations

$$
J_{0} \longrightarrow-J_{0}, \quad m_{B} \longrightarrow-m_{B}
$$

Thus the case of antiferromagnetic intersublattice interaction $J_{0}<0$ is completely equivalent to the case of ferromagnetic intersublattice interaction $-J_{0}>0$ by replacing $m_{B}$ by $-m_{B}$. This means that the $\mathrm{F}$ solution is replaced by the antiferromagnetic (AF) solution

$$
m_{A}=-m_{B}=m, \quad q_{A}=q_{B}=q .
$$

The results displayed in Fig. 1 remains valid, with $J_{0}$ replaced by $-J_{0}$ and $\mathrm{F}$ solution by AF solution.

\subsection{Non-zero applied field}

\subsubsection{Ferromagnetic intersublattice interaction}

In non-zero applied field $(H>0)$ and ferromagnetic intersublattice interactions $\left(J_{0}>0\right)$, only the paramagnetic $(\mathrm{P})$ solution is possible for the set of equations (17), which are of the form

$$
m_{A}=m_{B}=m>0, \quad q_{A}=q_{B}=q>0 .
$$

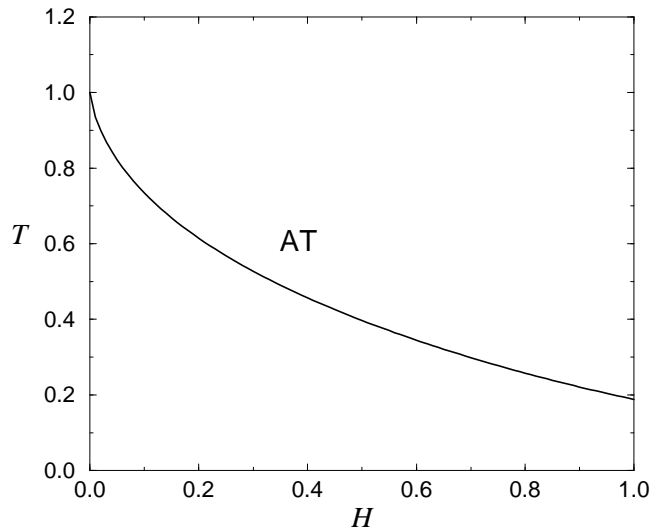

Figure 2. Region of stability in the presence of a field for the case of ferromagnetic intersublattice interaction $\left(J_{0}<0\right)$. The values of parameters are $J^{\prime} / J=1, J_{0}^{\prime} / J_{0}=1$ and $\left(J_{0}+\right.$ $\left.J_{0}^{\prime}\right) / \sqrt{J^{2}+J^{\prime 2}}=1 / 2$. There is only the $\mathrm{P}$ solution which is always L stable but becomes $\mathrm{T}$ unstable below the de AlmeidaThouless line AT. The temperature and field units in the axis are such that $\sqrt{J^{2}+J^{\prime 2}}=1$ and $k_{B}=1$.

This solution is always $\mathrm{L}$ stable, but becomes $\mathrm{T}$ unstable for low temperatures due to the violation of the condition (31), which in this case is also given by Eq. (58). The instability line is given by Eq. (59), illustrated in Fig. 2 for the case $J^{\prime} / J=1, J_{0}^{\prime} / J_{0}=1 / 2$ and $\left(J_{0}+J_{0}^{\prime}\right) / \sqrt{J^{2}+J^{\prime 2}}=2$. As should be expected, in the case $J_{0}=0$ and $J=0$, or $J_{0}^{\prime}=0$ and $J^{\prime}=0$, these results reduce to the de AlmeidaThouless line of one-sublattice SK model[5].

\subsubsection{Antiferromagnetic intersublattice interaction}

In non-zero applied field $(H>0)$ and antiferromagnetic intersublattice interactions $\left(J_{0}<0\right)$, two types of solutions to the set of equations (17) are possible:

- Paramagnetic (P) solution: $m_{A}=m_{B}=m>$ $0, q_{A}=q_{B}=q>0$.

- Antiferromagnetic (AF) solution: $m_{A} \neq m_{B}, q_{A} \neq$ $q_{B}$

For $\left(-J_{0}+J_{0}^{\prime}\right) / \sqrt{J^{2}+J^{\prime 2}} \leq 1 / 2$ only $\mathrm{P}$ solution is possible. This solution is always $\mathrm{L}$ stable but becomes $\mathrm{T}$ unstable at low temperatures due to the to the violation of the condition (31), which in this case it is also given by (58). The instability line is given by Eq. (59).

For $\left(-J_{0}+J_{0}^{\prime}\right) / \sqrt{J^{2}+J^{\prime 2}}>1 / 2$, AF solution also becomes possible. Fig. 3 shows the lines delimiting the regions of existence and stability of each type of solution for $-J_{0}^{\prime} / J_{0}=1 / 2$. The $\mathrm{P}$ solution becomes L unstable below line (a) due to the violation of the condition (49). In the case of $\mathrm{P}$ solution this condition is given by

$$
\begin{aligned}
L_{4}= & {\left[(1-q)(1-4 q+3 r)+2(t-m)^{2}\right]^{2}\left\{[ 1 - \beta ( J _ { 0 } ^ { \prime } + J _ { 0 } ) ( 1 - q ) ] \left[1-\beta\left(J^{\prime 2}-J^{2}\right)\right.\right.} \\
& \left.\times(1-4 q+3 r)]+2 \beta^{3}\left(J_{0}^{\prime}+J_{0}\right)\left(J^{\prime 2}-J^{2}\right)(t-m)^{2}\right\}\left\{\left[1-\beta\left(J_{0}^{\prime}-J_{0}\right)(1-q)\right]\right. \\
& \left.\times\left[1-\beta\left(J^{\prime 2}+J^{2}\right)(1-4 q+3 r)\right]+2 \beta^{3}\left(J_{0}^{\prime}-J_{0}\right)\left(J^{\prime 2}+J^{2}\right)(t-m)^{2}\right\}>0 .
\end{aligned}
$$


The first factor in (64) becomes negative inside line (a). Therefore the equation determining line (a) is

$$
\left[1-\beta\left(J_{0}^{\prime}-J_{0}\right)(1-q)\right]\left[1-\beta\left(J^{\prime 2}-J^{2}\right)(1-4 q+3 r)\right]+2 \beta^{3}\left(J_{0}^{\prime}-J_{0}\right)\left(J^{\prime 2}-J^{2}\right)(t-m)^{2}=0,
$$

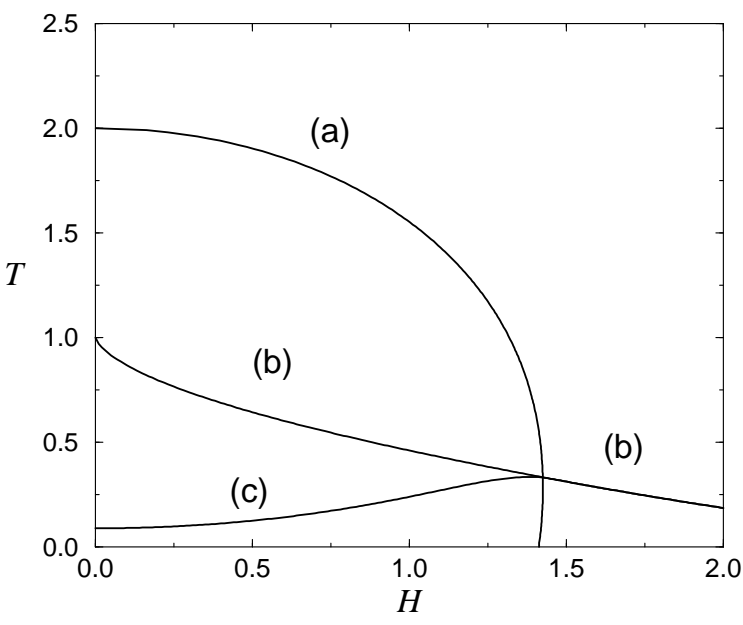

Figure 3. Regions of stability and existence of different solutions in the temperature versus field phase diagram for the case of antiferromagnetic intersublattice interaction $\left(J_{0}<0\right)$. The values of parameters are $J^{\prime} / J=1,-J_{0}^{\prime} / J_{0}=1 / 2$ and $\left(-J_{0}+\right.$ $\left.J_{0}^{\prime}\right) / \sqrt{J^{2}+J^{\prime 2}}=2$. The $\mathrm{P}$ solution is $\mathrm{L}$ stable outside line (a) and $\mathrm{T}$ stable above line (b). The AF solution is possible only inside line (a) and it is always $\mathrm{L}$ stable, but becomes $\mathrm{T}$ unstable below line (c). The temperature and field units in the axis are such that $\sqrt{J^{2}+J^{\prime 2}}=1$ and $k_{B}=1$.

which is in agreement with previous study [12]. The P solution is $\mathrm{T}$ unstable below line (b). This instability occurs due to the violation of condition (31), given in this case by Eq. (58), caused by the second factor. Therefore the line (b) is determined by equation (59). The AF solution is possible only inside line (a). It is L stable throughout this region and $\mathrm{T}$ unstable below line (c). This instability is due to the violation of condition (31). Therefore line (c) obeys the equation

$$
\begin{gathered}
{\left[1-\left(\beta J^{\prime}\right)^{2}\left(1-2 q_{A}+r_{A}\right)\right]\left[1-\left(\beta J^{\prime}\right)^{2}\left(1-2 q_{B}+r_{B}\right)\right]} \\
=(\beta J)^{4}\left(1-2 q_{A}+r_{A}\right)\left(1-2 q_{B}+r_{B}\right)
\end{gathered}
$$

Rejecting solutions that are $\mathrm{L}$ unstable, we conclude that $\mathrm{P}$ phase exists outside and AF phase inside line (a). The P solution becomes $\mathrm{T}$ unstable below line (b) and AF solution below line (c), which meet smoothly on the line (a). In the region below lines (b) and (c) it is necessary to consider replica symmetry breaking solution, which will presumably change line (a) in this region.

For sufficiently large values of the ratio $-J_{0}^{\prime} / J_{0}$ the model can exhibit first-order transition from AF phase to the $\mathrm{P}$ phase [12]. As an example, we consider the case $-J_{0}^{\prime} / J_{0}=5$ shown in Fig. 4. The $\mathrm{P}$ solution is $\mathrm{L}$ stable inside line (a) given by Eq. (65), and T stable above line (b) given by Eq. (66). There is one AF solution inside line (a) and two distinct AF solutions between lines (a) and (d), as illustrated in Figs. 5(a) and 5(b) for $k_{B} T / \sqrt{J^{2}+J^{\prime 2}}=1$. One of the AF solutions, corresponding to dotted lines in Fig. 5, is L unstable due to the violation of the condition (49), as shown in Fig. 5(c). The transition between AF phase and $\mathrm{P}$ phase is first order, determined by equating the free energies of $\mathrm{L}$ stable $\mathrm{AF}$ and $\mathrm{P}$ phases, as shown in Fig. 5(d). The first-order transition line is shown as dotted line in Fig. 4, which ends at the tricritical point TCP. The L stable AF solution becomes T unstable below line (c) due to the violation of condition (31), and it is determined by Eq. (66). We conclude that in Fig. 4 the P phase exists outside and AF phase inside lines (a) and (e), which meet smoothly at the tricritical point TCP. The $\mathrm{P}$ phase becomes $\mathrm{T}$ unstable below line (b), and AF phase below line (c). Notice that the lines (b) and (c) are discontinuous across first-order transition [12]. It is likely that the first-order transition line will change in this part of phase diagram once the replicasymmetry-breaking solutions are considered for $\mathrm{P}$ and $\mathrm{AF}$ phases.

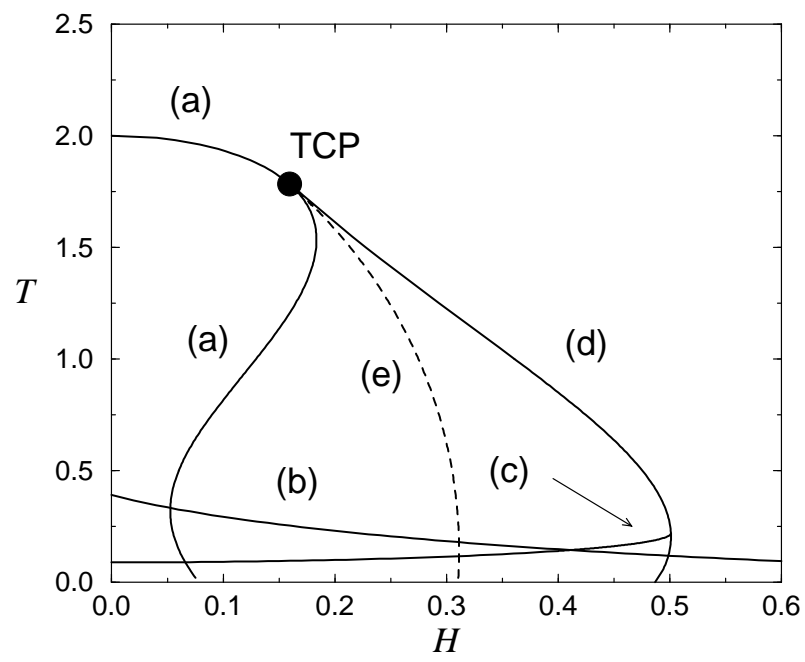

Figure 4. Regions of stability and existence of different solutions in the temperature versus field phase diagram for the case of antiferromagnetic intersublattice interaction $\left(J_{0}<0\right)$. The values of parameters are $J^{\prime} / J=1,-J_{0}^{\prime} / J_{0}=5$ and $\left(-J_{0}+\right.$ $\left.J_{0}^{\prime}\right) / \sqrt{J^{2}+J^{\prime 2}}=2$. The $\mathrm{P}$ solution is $\mathrm{L}$ stable outside the line (a) and T stable above line (b). There is one AF solution inside line (a) which is always L stable, and two distinct AF solutions between lines (a) and (d), one L stable and the other $\mathrm{L}$ unstable. The L stable AF solution is also T stable above line (c). The line (e) is the first-order transition line and TCP is the tricritical point. The temperature and field units in the axis are such that $\sqrt{J^{2}+J^{\prime 2}}=1$ and $k_{B}=1$. 

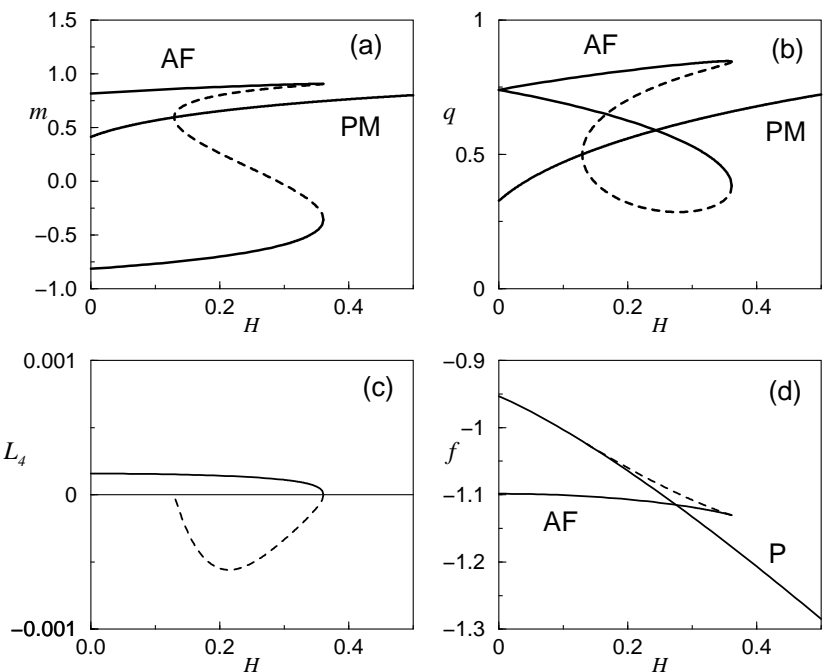

Figure 5. Field behavior of various quantities for the fixed value of temperature $k_{B} T / \sqrt{J^{2}+J^{\prime 2}}=1$ in the phase diagram of Fig. 4 . In (a) and (b) the order parameters of the $\mathrm{P}$ and $\mathrm{AF}$ solution are shown, in (c) the L stability condition $L_{4}$ of the AF solution, and finally in (d) the free-energy per spin of $\mathrm{P}$ and AF solutions. The $\mathrm{L}$ unstable AF solution are represented by dotted lines, and corresponds to the upper portion of the van der Waals loop in (d). The first-order transition is determined by the intersection of $\mathrm{L}$ stable $\mathrm{AF}$ and $\mathrm{P}$ solutions, as depicted in (d). The temperature and energy units in the axis are such that $\sqrt{J^{2}+J^{\prime 2}}=1$ and $k_{B}=1$.

\section{Conclusions}

In this paper we have investigated the stability of the RS symmetric solution of the two-sublattice generalization of the SK infinite-range spin-glass model. We have derived stability conditions for transversal fluctuations in agreement with previous investigations, and we have extended previous study of the stability against longitudinal or anomalous fluctuations. The eigenvalues associated with such perturbations are in general complex. We generalized the usual stability condition by requiring the real part of these eigenvalues to be positive. The necessary and sufficient stability conditions were found using the Hurwitz criterion for all the roots of the secular equation to have positive real parts. These conditions allowed us to select one RS solution among those that are transversally stable. We believe that the generalized stability condition should also be useful in other spin glass models where eigenvalues associated with longitudinal and anomalous perturbations become complex.

\section{Acknowledgments}

The authors acknowledge partial financial support from the Brazilian Government Agencies PROCAD/CAPES and CNPq.

\section{References}

[1] D. Sherrington and S. Kirkpatrick, Phys. Rev. Lett. 35, 1792 (1975).

[2] K. Binder and A. P. Young, Rev. Mod. Phys. 58, 801 (1986).

[3] M. Mézard, G. Parisi, and M. A. Virasoro, Spin Glass Theory and Beyond (World Scientific, Singapore, 1987).

[4] K. H. Fischer and J. H. Hertz, Spin Glasses (Cambridge University Press, Cambridge, 1991).

[5] J. R. L. de Almeida and D. J. Thouless, J. Phys. A 11, 983 (1978).

[6] G. Parisi, Phys. Lett. 73A, 203 (1979).

[7] G. Parisi, J. Phys. A 13, L115 (1980).

[8] G. Parisi, J. Phys. A 13, 1101 (1980).

[9] G. Parisi, J. Phys. A 13, 1887 (1980).

[10] I. Y. Korenblit and E. F. Shender, Zh. Eksp. Teor. Fiz. 89, 1785 (1985), [Sov. Phys. JETP 62, 1030 (1985)].

[11] Y. V. Fyodorov, I. Y. Korenblit, and E. F. Shender, J. Phys. C 20, 1835 (1987).

[12] Y. V. Fyodorov, I. Y. Korenblit, and E. F. Shender, Europhys. Lett. 4, 827 (1987).

[13] H. Takayama, Prog. Theor. Phys. 80, 827 (1988).

[14] D. Bertrand, A. R. Fert, M. C. Schmidt, F. Bensamka, and S. Legrand, J. Phys. C 15, L883 (1982).

[15] P. zen Wong, S. von Molnar, T. T. M. Palstra, J. A. Mydosh, H. Yoshizawa, S. M. Shapiro, and A. Ito, Phys. Rev. Lett. 55, 2043 (1985).

[16] P. zen Wong, H. Yoshizawa, and S. M. Shapiro, J. Appl. Phys. 57, 3462 (1985).

[17] H. Yoshizawa, S. Mitsuda, H. Aruga, and A. Ito, Phys. Rev. Lett. 59, 2364 (1987).

[18] H. Yoshizawa, S. Mitsuda, H. Aruga, and A. Ito, J. Phys. Soc. Jpn. 58, 1416 (1989).

[19] H. Yoshizawa, H. Mori, H. Kawano, H. Aruga-Katori, S. Mitsuda, and A. Ito, J. Phys. Soc. Jpn. 63, 3145 (1994).

[20] C. de Dominicis and I. Kondor, in Applications of Field Theory to Statistical Mechanics, Vol. 216 of Lecture Notes in Physics, edited by L. Garrido (Springer-Verlag, Berlin, 1985), pp. 91-106.

[21] E. J. S. Lage and J. R. L. de Almeida, J. Phys. C 15, L1187 (1982).

[22] F. A. da Costa, C. S. O. Yokoi, and S. R. A. Salinas, J. Phys. A 27, 3365 (1994).

[23] J. V. Uspensky, Theory of Equations (McGraw-Hill Book Company, Inc., New York, 1948).

[24] G. Toulouse, J. Phys. (Paris) Lett. 41, L (1980). 